\title{
Convenient Access to Hydroquinone and Quinone Derivatives from Cyclobutenedione Units
}

\author{
Thanh V. Nguyen ${ }^{\mathrm{A}}$ \\ A Research School of Chemistry, Australian National University, Canberra ACT 0200, Australia. \\ Email: thanh@rsc.anu.edu.au
}

Manuscript received: 11 May 2010.

Manuscript accepted: 25 June 2010.

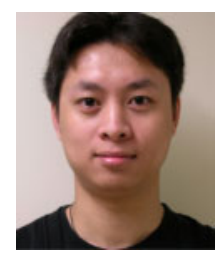

Thanh V. Nguyen graduated from University of New South Wales with B.Sc.(Hons). In 2006, he commenced study towards his Ph.D. at the Australian National University under the supervision of Associate Professor Michael S. Sherburn. He has been working on the design, synthesis, and guest binding studies of new synthetic hosts. He has recently submitted his doctoral thesis and is going to move to Germany to pursue research in organocatalysis.

An important synthetic method providing access to a variety of heterocyclic and polycyclic quinones and hydroquinones is described. The key transformation involves the thermal ring expansion of cyclobutenedione derivatives to quinone/hydroquinone units. The synthetic utility and recent applications of this method in natural product synthesis are also discussed.

\section{Introduction}

Thirty years ago, cyclobutenediones were only studied as theoretically interesting molecules without much thought towards their potential use in building complex structures. ${ }^{[1-3]}$ Late in the 1980 s, these compounds started to find their way into synthetic organic chemistry via their conversion to highly functionalized benzoquinones and cyclopentenones. ${ }^{[1]}$ During the studies of unsaturated ketenes, ${ }^{[4,5]}$ Moore and coworkers discovered one of the most important uses of cyclobutenediones while carrying out the thermolysis of 4-alkynyl-4-hydroxycyclobutenones. These compounds were able to undergo $4 \pi$-electrocyclic ring opening to the corresponding enynylketenes, which subsequently ring close to form diradical intermediates. These intermediates then rearrange to form quinone or hydroquinone derivatives via either radical addition or homolytic substitution, offering a convenient synthetic method to access polycyclic compounds containing quinone/hydroquinone moieties from cyclobutenediones. ${ }^{[4-20]}$

\section{Thermal Ring Expansion of 4-Alkynyl-4-Hydroxycyclobutenones}

In preliminary versions of this transformation, ${ }^{[4,5]}$ simple 4-alkynyl-4hydroxycyclobutenones were converted to quinone derivatives (Scheme 1) First, the readily available cyclobutenediones ${ }^{[1,21-23]} \mathbf{1}$ were transformed into 4-alkynyl-4-hydroxycyclobutenones 2 by straightforward nucleophilic addition to one of the carbonyl groups. ${ }^{[6-9,12-16,18]} \mathbf{2}$ were then heated with or without transition metal catalysts to perform a ring expansion-cyclization to produce the highly functionalized quinones $\mathbf{5}^{[4,5]}$ which could be easily reduced to give hydroquinone derivatives. This process was later proved to be a cascade reaction involving several highly reactive intermediates: the conjugated ketene $\mathbf{3}$ and the benzenoid diradical 4 (Scheme 1). ${ }^{[6-19]}$

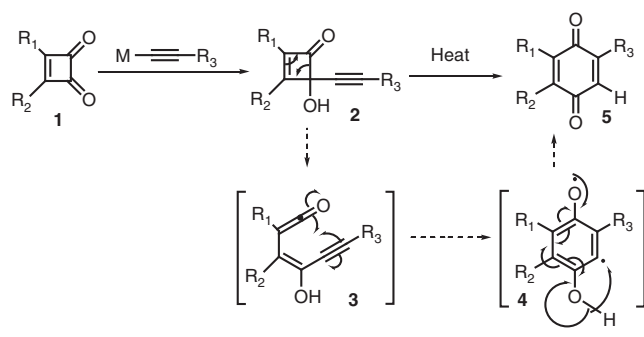

Scheme 1. Quinone derivatives from cyclobutenediones.

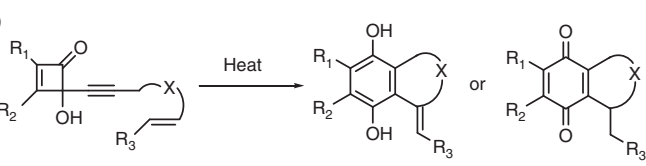

(b)<smiles>[R]CCC#CC1(OC#C[2H])C(=O)C([2H])=C1[R7]</smiles><smiles>[R]C=Cc1c(O)c([R])c([2H])c2c1CCO2</smiles>

(c)
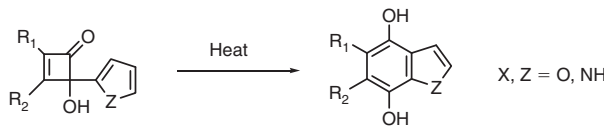

Scheme 2. Polycyclic and heterocyclic compounds from cyclobutenediones.

\section{Recent Applications}

The thermal ring expansion of substituted cyclobutenones to quinone and hydroquinone derivatives has recently been employed in numerous syntheses of natural products and bioactive compounds. ${ }^{[7,8,12,14,18,24,25]}$ Alkaloids assoanine ${ }^{[14]}$ and cribrostatin $6^{[24]}$ along with diterpenes (-)-colombiasin $A^{[25]}$ and (-)elisapterosin $\mathrm{B}^{[25]}$ (Scheme 3 ) are selected examples of a potentially broad class of such naturally occurring compounds that could be prepared by this method This effective and convenient transformation, termed Moore rearrangement, will be among the first choices for future synthetic strategies of biologically valuable organic compounds containing quinone/hydroquinone moieties. ${ }^{[26,27]}$
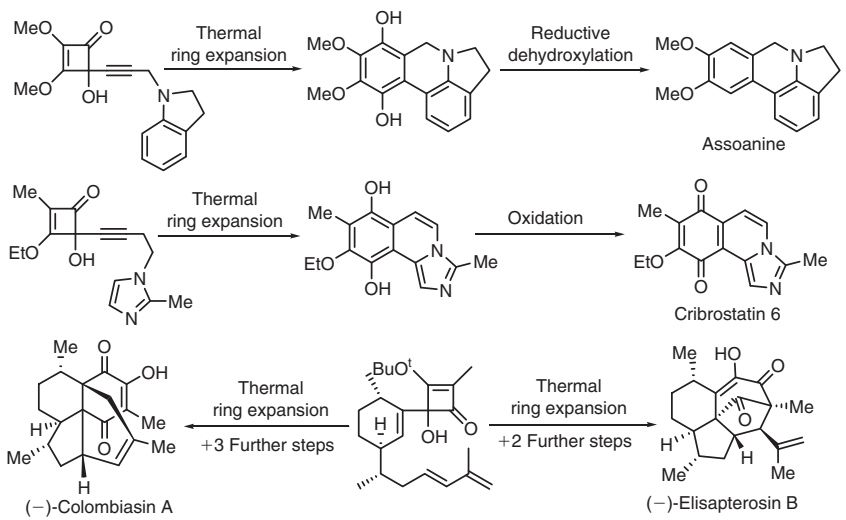

Scheme 3. Examples of natural products synthesized using the Moore rearrangement. 


\section{References}

[1] L. S. Liebeskind, Tetrahedron 1989, 45, 3053. doi:10.1016/S0040-4020(01)80131-9

2] A. H. Schmidt, W. Reid, Synthesis 1978, 1. doi:10.1055/S-1978-24652

3] H. Knorr, W. Reid, Synthesis 1978, 649. doi:10.1055/S-1978-24841

[4] J. O. Karlsson, N. V. Nguyen, L. D. Foland, H. W. Moore, J. Am. Chem. Soc. 1985, 107, 3392. doi:10.1021/JA00297A081

[5] S. T. Perri, L. D. Foland, O. H. W. Decker, H. W. Moore, J. Org. Chem. 1986, 51, 3067. doi:10.1021/JO00365A044

[6] O. H. W. Decker, H. W. Moore, J. Org Chem 1987, 52 , 1174 doi:10.1021/JO00382A045

[7] M. W. Reed, H. W. Moore, J. Org. Chem. 1988, 53, 4166 doi:10.1021/JO00253A002

[8] L. D. Foland, O. H. W. Decker, H. W. Moore, J. Am. Chem. Soc. 1989, 111, 989. L. D. Foland, O. H. W.
doi: $10.1021 / \mathrm{JA} 00185 \mathrm{~A} 031$

[9] S. T. Perri, H. W. Moore, J. Am. Chem. Soc. 1990, 112, 1897. doi:10.1021/JA00161A039

[10] S. L. Xu, M. Taing, H. W. Moore, J. Org. Chem. 1991, 56, 6104. doi:10.1021/JO00021A02

[11] H. Xia, H. W. Moore, J. Org. Chem. 1992, 57, 3765. doi:10.1021/JO00040A008

[12] H. Liu, L. M. Gayo, R. W. Sullivan, A. Y. H. Choi, H. W. Moore, J. Org. Chem. 1994, 59, 3284. doi:10.1021/JO00091A013

[13] Y. Xiong, H. Xia, H. W. Moore, J. Org. Chem. 1995, 60, 6460. doi:10.1021/JO00125A037

[14] Y. Xiong, H. W. Moore, J. Org. Chem. 1996, 61, 9168. doi:10.1021/JO9613803

[15] M. J. Heileman, R. Tiedemann, H. W. Moore, J. Am. Chem. Soc. 1998, 120, 3801. doi: $10.1021 / \mathrm{JA} 980039 \mathrm{~S}$
[16] R. Tiedemann, M. J. Heileman, H. W. Moore, J. Org. Chem. 1999, 64, 2170. doi:10.1021/ JO982422L

[17] R. Tiedemann, P. Turnbull, H. W. Moore, J. Org. Chem. 1999, 64, 4030. doi:10.1021/ JO990052A

[18] A. R. Hergueta, H. W. Moore, J. Org. Chem. 1999, 64, 5979. doi:10.1021/JO990505B

[19] A. R. Hergueta, H. W. Moore, J. Org. Chem. 2002, 67, 1388. doi:10.1021/JO010919G

[20] P. Wipf, C. R. Hopkins, J. Org. Chem. 1999, 64, 6881. doi:10.1021/JO990089V

[21] M. W. Reed, D. J. Pollart, S. T. Perri, L. D. Foland, H. W. Moore, J. Org. Chem. 1988, 53, 2477. doi: $10.1021 / \mathrm{JO} 00246 \mathrm{~A} 016$

[22] H. Liu, C. S. Tomooka, H. W. Moore, Synth. Commun. 1997, 27, 2177. doi:10.1080 00397919708006826

23] A. Mukkanti, M. Periasamy, Arkivoc 2005, 11, 48

24] D. Knueppel, S. F. Martin, Angew. Chem. Int. Ed. 2009, 48, 2569. doi:10.1002/ANIE. 200806269

[25] D. C. Harrowven, D. D. Pascoe, D. Demurtas, H. O. Bourne, Angew. Chem. Int. Ed. 2005, 44 1221. doi:10.1002/ANIE.200462268

[26] J. C. Namyslo, D. E. Kaufmann, Chem. Rev. 2003, 103, 1485. doi:10.1021/CR010010Y

[27] K. C. Nicolaou, D. J. Edmonds, P. G. Bulger, Angew. Chem. Int. Ed. 2006, 45, 7134 doi:10.1002/ANIE. 200601872 